\title{
Impacto na redução da hemoglobina glicada com ampliação do acesso às medicações para o tratamento do diabetes tipo 2
}

\author{
Impact on the reduction of glycated hemoglobin with increased access to medications for \\ the treatment of type 2 diabetes
}

\begin{abstract}
Impacto en la reducción de la hemoglobina glucosilada con un mayor acceso a medicamentos para el tratamiento de la diabetes tipo 2
\end{abstract}

Paulo Henrique de Melo Filho ${ }^{1 *}$, José Maria Correia Lima e Silva ${ }^{1}$, Pryscilla Oliveira Melo².

\begin{abstract}
RESUMO
Objetivo: Avaliar o controle glicêmico em diabéticos do tipo 2 acompanhados no ambulatório de endocrinologia de um hospital terciário do Nordeste em uso de antidiabéticos orais e insulinas dispensadas pela farmácia de medicamentos especializados. Métodos: Estudo observacional e transversal. As variáveis consideradas foram o nível de hemoglobina glicada ( $\mathrm{HbA1c})$, sexo, idade, procedência, tempo de diagnóstico, tempo de tratamento, frequência de consultas por ano, classe de medicamentos utilizado e presença de comorbidades. Os dados foram analisados no software $R$ versão 3.5.1. A amostra foi caracterizada por frequências absolutas e percentuais, média e desvio padrão, e utilizados teste de Anderson-Darling, Levene, t de Student, $t$ de Student pareado, McNemar, qui-quadrado de Pearson e, por fim, foi calculada a razão de chances (odds ratio). Resultados: Analisados 130 pacientes, 95 aptos a participar da pesquisa, 62,1\% do sexo feminino. Frequência inicial de controle glicêmico adequado era de $15 \%$ e após acompanhamento passou para 48\%. Média inicial de $\mathrm{HbA1c}$ era de 8,6 $\pm 1,60$ e após 7,4 $\pm 1,65$. Conclusão: Pacientes analisados obtiveram melhor controle comparando o início com o atual momento, evidenciado tanto pelo aumento do número de pacientes com $\mathrm{HbA} 1 \mathrm{c} \leq 7$, como pela redução da média de hemoglobina glicada.
\end{abstract}

Palavras-chave: Diabetes Mellitus, Controle glicêmico, Hemoglobina glicada.

\begin{abstract}
Objective: Evaluate glycemic control in type 2 diabetics followed at the endocrinology outpatient clinic of a tertiary hospital in the Northeast in the use of oral antidiabetic agents and insulin dispensed by the specialized drugstore. Methods: Observational and transversal study. The variables considered were the level of glycated hemoglobin $(\mathrm{HbA} 1 \mathrm{c})$, sex, age, origin, diagnosis time, treatment time, the frequency of visits per year, class of medications used and presence of comorbidities. The data were analyzed in the R software version 3.5.1. The sample was characterized by absolute and percentage frequencies, mean and standard deviation, and used the Anderson-Darling test, Levene, Student $t$, Student t paired, McNemar, Pearson's chi-square, and finally the ratio odds was calculated. Results: A total of 130 patients were analyzed, 95 were eligible to participate in the study, $62.1 \%$ were female. The initial frequency of adequate glycemic control was $15 \%$ and after followup was increased to $48 \%$. The initial mean $\mathrm{HbA1c}$ was $8.6 \pm 1.60$ and after $7.4 \pm 1.65$. Conclusion: Patients analyzed had a better control comparing the onset with the present moment, evidenced by both the increase in the number of patients with $\mathrm{HbA} 1 \mathrm{c} \leq 7$ and the reduction in the mean of glycated hemoglobin.
\end{abstract}

Keywords: Diabetes Mellitus, Glycemic control, Glycated hemoglobin.

1 Universidade Federal do Piauí (UFPI), Teresina - PI. *E-mail: paulo_mello@homail.com

2 Centro Universitário do Maranhão (UNICEUMA), São Luís - MA. 


\section{RESUMEN}

Objetivo: Evaluar el control glucémico en diabéticos tipo 2 seguidos en el ambulatorio de endocrinología de un hospital terciario en el Noreste utilizando antidiabéticos orales e insulina dispensada por la farmacia especializada en medicamentos. Métodos: Estudio observacional y transversal. Las variables consideradas fueron el nivel de hemoglobina glucosilada ( $\mathrm{HbA1c})$, sexo, edad, origen, tiempo desde el diagnóstico, tiempo de tratamiento, frecuencia de consultas por año, clase de medicación utilizada y presencia de comorbilidades. Los datos se analizaron utilizando el software $R$ versión 3.5.1. La muestra se caracterizó por frecuencias absolutas y porcentuales, media y desviación estándar, y se utilizaron Anderson-Darling, Levene, t de Student, t de Student pareada, McNemar, chi-cuadrado de Pearson y finalmente se calculó la razón. Resultados: Se analizaron 130 pacientes, 95 capaces de participar en la investigación, 62,1\% mujeres. La frecuencia inicial de control glucémico adecuado fue del $15 \%$ y después del seguimiento aumentó al $48 \%$. La media inicial de $\mathrm{HbA} 1 \mathrm{c}$ fue de 8,6 $\pm 1,60$ y después de 7,4 $\pm 1,65$. Conclusión: Los pacientes analizados tuvieron mejor control comparando el inicio con el momento actual, evidenciado tanto por el aumento del número de pacientes con $\mathrm{HbA} 1 \mathrm{c} \leq 7$ como por la reducción de la hemoglobina glucosilada media.

Palabras clave: Diabetes Mellitus, Control glucémico, Hemoglobina glucosilada.

\section{INTRODUÇÃO}

O Diabetes Melito (DM) se caracteriza por diferentes etiologias de um grupo de doenças metabólicas, no qual o principal mecanismo fisiopatológico é a hiperglicemia que ocorre em decorrência de uma secreção deficiente de insulina pelas células beta, resistência periférica à ação da insulina ou ambas (AMERICAN DIABETES ASSOCIATION (ADA), 2020).

A Sociedade Brasileira de Diabetes (SBD), assim como outras sociedades de diabetes no mundo acompanham a Organização Mundial de Saúde (OMS) que classifica o DM com base em sua etiologia. O DM tipo 1 é decorrente da destruição das células beta-pancreáticas, ocasionando deficiência completa na produção de insulina. O DM tipo 2 é definido pela perda progressiva de secreção insulínica associada a resistência à insulina. O DM gestacional se caracteriza por hiperglicemia de graus variados diagnosticada durante a gestação, na ausência de critérios de DM prévio. Os outros tipos de DM são caracterizados por defeitos na secreção de insulina, por defeitos na ação da insulina, monogênicos (Maturity Onset Diabetes of Young (MODY), diabetes neonatal, secundário a endocrinopatias, secundário a doenças do pâncreas exócrino, secundário a infecções, secundário a medicamentos e associado a outras doenças genéticas (SBD, 2021).

Para o diagnóstico de DM devem ser considerados os critérios preconizados pela SBD, em acordo com a OMS e a ADA. O diagnóstico estabelecido é observado com valores de glicemia de jejum maior ou igual a $126 \mathrm{mg} / \mathrm{dL}$ em mais de uma ocasião ou glicemia de duas horas no Teste Oral de Tolerância à Glicose (TOTG) maior ou igual a $200 \mathrm{mg} / \mathrm{dL}$ ou hemoglobina glicada (HbA1c) maior ou igual a 6,5\%. Também é possível se estabelecer o diagnóstico com a determinação ocasional da glicemia plasmática, independente do jejum, com resultado maior ou igual 200 desde que na presença de sintomas inequívocos de hiperglicemia, tais como poliúria, polidipsia, polifagia e perda de peso, não havendo necessidade de confirmação por meio de segunda dosagem (SBD, 2021).

A Federação Internacional do Diabetes (IDF), levando em consideração a população com idade entre 20 e 79 anos estimada pela Divisão de População das Nações Unidas, calcula que o número de pessoas com diabetes no mundo era de 463 milhões de pessoas, sendo que metade desconhecem que têm a condição e, portanto, correm alto risco de desenvolver complicações graves relacionadas ao diabetes. Além disso, 0 impacto econômico em decorrência do diabetes é bastante relevante, visto que três em cada quatro pessoas com o diagnóstico, cerca de 352 milhões de pessoas, estão em idade economicamente ativa (ou seja, entre 20 e 64 anos). Isso gera um impacto humano crescente, culminando em redução da produtividade e do crescimento econômico nas próximas décadas. No Brasil, o número de pessoas com diabetes foi de 16,8 
milhões. Segundo estimativas para o ano de 2045, haverá 700 milhões de pessoas com diabetes no mundo e no Brasil esse número pode alcançar mais de 26 milhões (INTERNATIONAL DIABETES FEDERATION (IDF), 2019).

O Estudo Brasileiro sobre os Custos do Diabetes (ESCUDI), realizado com o objetivo de estimar os custos do tratamento do diabetes no Brasil, evidenciou que o custo total por ano para atendimento ambulatorial é de 2.108 dólares por paciente, dos quais 1.335 de custos diretos e 773 de custos indiretos. A maior parcela dos custos diretos foi atribuída à medicação e os custos aumentaram juntamente com a duração da doença, o nível de cuidado e a presença de complicações crônicas microvasculares e macrovasculares, o que sugeriu a necessidade de realocar recursos de saúde com foco na prevenção primária do diabetes e de suas complicações (BAHIA LR, et al., 2011).

A fim de evitar complicações, o tratamento eficaz para o controle glicêmico adequado deve ser assegurado ao paciente diabético, visto que as leis 8080/90 e 8142/90, através dos princípios da descentralização dos serviços de saúde e municipalização da gestão, destacando o artigo $6^{\circ}$ da Lei $n^{\circ} 8080$ mostram que o Sistema Único de Saúde (SUS) tem como dever fornecer os suprimentos e os medicamentos necessários para a adequada assistência terapêutica integral, inclusive a assistência farmacêutica (BRASIL, 1990).

Em 2002, a Portaria no 371/GM criou o Programa Nacional de Assistência Farmacêutica para Diabetes Mellitus que tem como objetivo ofertar de maneira contínua para a rede básica de saúde os medicamentos metformina $850 \mathrm{mg}$, glibenclamida $5 \mathrm{mg}$ e insulinas (regular e NPH), definidos e propostos pelo Ministério da Saúde, validados e pactuados pelo Comitê do Plano Nacional de Reorganização da Atenção a Hipertensão Arterial e Diabetes (BRASIL, 2002). Recentemente, a Portaria $\mathrm{n}^{\circ} \mathbf{1 . 0 9 6}$, de 25 de novembro de 2020, inclui medicamento Dapaglifozina $10 \mathrm{mg}$ como pertencente ao Componente Especializado da Assistência Farmacêutica (CEAF), da Tabela de Procedimentos, Medicamentos, Órteses, Próteses e Materiais Especiais do SUS., porém com uma série de critérios pré-estabelecidos para o fornecimento, incluindo a necessidade de um evento cardiovascular prévio, e não para todos os pacientes com diabetes (BRASIL, 2020).

No estado do Piauí, a Diretoria de Unidade de Assistência Farmacêutica é responsável pelo planejamento, aquisição, organização, armazenamento, distribuição e controle de medicamentos para atender diversos programas, entre eles: Programas Estratégicos, Componente Especializado (Excepcional - Alto custo), Atenção Básica e Hospitalar. As medicações disponibilizadas através do Laudo de solicitação, avaliação e autorização de medicamentos (LME) para o tratamento do Diabetes mellitus tipo 1 e tipo 2 (CID-10 E 10.0 e E 11.0) são: Inibidores da dipeptidil peptidase 4 (DDP-4), como a Saxagliptina (2,5 e $5 \mathrm{mg}$ ), Vidagliptina (50 $\mathrm{mg}$ ) e Sitagliptina (50 e $100 \mathrm{mg}$ ), também em associação com Metformina na dosagem de 500, 850 e 1000 mg; Glitazonas, como a Pioglitazona (15, 30 e 45mg) e análogos de insulina de ação rápida (Glulisina, Asparte e Lispro), ação intermediária (Detemir) e ação longa (Glargina) (PIAUÍ, 2020).

Estudos clássicos conduzidos em pacientes com diagnóstico de diabetes tipo 2 e tipo 1, como o United Kingdom Prospective Diabetes Study (UKPDS, 1998) e o Diabetes Control and Complications Trial (DCCT, 1993), tiveram como objetivo demonstrar que o tratamento do paciente com DM se baseia no bom controle metabólico, diminuindo, assim, os riscos de complicações micro e macrovasculares, tendo em vista que o controle da glicemia reduz de forma significativa as complicações da doença e que o descontrole permanente acarreta, no decorrer dos anos, uma série de complicações orgânicas, resultando em danos teciduais, perda de função e falência de vários órgãos. Esses estudos mostraram que a meta para o tratamento da hiperglicemia no diabetes tipo 2 é atingir níveis de $\mathrm{HbA1c}$ menores que 7\%, glicemia de jejum entre 70-130 $\mathrm{mg} / \mathrm{dL}$ e pós-prandial menor que $180 \mathrm{mg} / \mathrm{dL}$ (BERTOLUCI MC, et al., 2014)

Diante do exporto e da crescente necessidade de ampliar o arsenal terapêutico para o paciente com diabetes no âmbito do SUS, visando um adequado controle glicêmico, a fim de reduzir complicações vasculares, o objetivo desse estudo foi avaliar a frequência do controle glicêmico adequado e fatores associados, pela avaliação dos níveis de hemoglobina glicada, em pacientes com DM tipo 2 acompanhados no ambulatório de endocrinologia de um hospital terciário do Nordeste em uso de antidiabéticos orais e insulinas dispensadas pela farmácia de medicamentos especializados do Piauí no período de junho a setembro de 2018. 


\section{MÉTODOS}

Trata-se de estudo observacional e transversal, realizado no ambulatório de Endocrinologia e Metabologia de um hospital terciário do Nordeste e na Farmácia de Medicamentos Especializados do Piauí, através da análise dos prontuários. Foram incluídos todos os prontuários de pacientes com o diagnóstico de diabetes acompanhados no referido ambulatório e que solicitaram medicação através do CID-10 E10.0 e E11.0 na Farmácia de Medicamentos Especializados do Piauí através do Laudo de solicitação, avaliação e autorização de medicamentos (LME) no período de junho a setembro de 2018, com idade maior ou igual a 18 anos. Vale ressaltar que o período utilizado é referente ao tempo necessário para a renovação do LME e dispensação da medicação utilizada. Foram excluídos pacientes com diagnóstico de diabetes tipo 1, aqueles que solicitaram a medicação pela primeira vez e prontuários incompletos.

O controle glicêmico foi categorizado conforme o nível de HbA1c, considerado adequado quando menor ou igual a $7 \%$ e inadequado quando maior que $7 \%$. As variáveis associadas foram expressas nas seguintes categorias: sexo, idade, tempo de diagnóstico, tempo de uso do medicamento dispensado por LME, frequência de consultas por ano e classe de medicamentos utilizada.

As informações sobre variáveis foram coletadas através de revisão de prontuários com auxílio da ficha de coleta de dados e submetidos a processo de digitação, utilizando-se planilhas do aplicativo Microsoft Excel e, posteriormente, exportados e analisados no software $R$ versão 3.5.1.

A amostra foi caracterizada por meio de frequências absolutas e relativas, assim como por meio das estatísticas descritivas: média e desvio padrão. O teste de Anderson-Darling foi aplicado as variáveis numéricas para verificação do pressuposto de normalidade. A homogeneidade das variâncias foi avaliada pelo teste de Levene.

$\mathrm{Na}$ análise bivariada, as comparações entre grupos independentes de variáveis numéricas foram feitas por meio do teste $t$ de Student e entre grupos dependentes de variáveis numéricas por meio do teste $t$ de Student pareado; a homogeneidade de grupos dependentes de variáveis categóricas foi testada por meio do teste de McNemar e entre grupos independentes por meio do teste qui-quadrado de Pearson e, por fim, foi calculada a razão de chances (odds ratio) e seu respectivo intervalo de confiança de $95 \%$ para os grupos tiveram o teste qui-quadrado estatisticamente significativo. Para todas as análises foi adotado o nível de significância de $5 \%$.

Foi solicitado, mediante a assinatura, pelo paciente e/ou seu responsável legal, do Termo de Consentimento Livre e Esclarecido, autorizando a realização da pesquisa, bem como a divulgação dos resultados. Para manter a confidencialidade dos pacientes incluídos na pesquisa foi realizada uma codificação numérica aleatoriamente, de modo que o nome e os demais dados de prontuário fossem preservados em sigilo. Tendo em vista tratar-se de estudo com coleta de dados em registros de prontuários médicos, nos casos em que a obtenção do TCLE não se tornou possível de forma presencial, foi solicitada dispensa do uso do Termo de Consentimento Livre e Esclarecido ao CEP. O trabalho foi aprovado pelo Comitê de Ética e Pesquisa (CEP), vinculado ao Hospital Universitário da Universidade Federal do Piauí, sob CAAE 94049118.6.0000.8050.

\section{RESULTADOS}

No período de junho a setembro de 2018, a amostra totalizou 130 pacientes, dentre os quais 95 aptos a participar da pesquisa. Foram excluídos 35 , sendo 4 com diagnóstico de diabetes tipo 1, 28 por ser a primeira solicitação por LME e 3 em razão de dados incompletos nos prontuários.

Dos 95 prontuários analisados, 36 pacientes eram do sexo masculino e 59 do sexo feminino. A idade média dos pacientes foi de $61,2 \pm 12,38$ anos variando de 24 a 86 anos. A maioria dos pacientes $(60 \%)$ tem tempo de doença maior que 10 anos; $20 \%$ tempo de doença menor do que 5 anos e, também, $20 \%$ tempo de doença entre 5 e 10 anos. A frequência de consulta foi superior a quatro vezes por ano em mais da metade dos pacientes. 
No momento de iniciar o processo para recebimento da medicação por LME, a frequência inicial de pacientes com controle glicêmico adequado $(\mathrm{Hb} 1 \mathrm{Ac} \leq 7)$ era de $15 \%$ e após o tratamento com as medicações disponibilizadas passou a ser de $48 \%$ ( $p$-valor $<0,001$ ). A princípio, a média da HbA1c dos pacientes era de $8,6 \pm 1,60$ e após passou a ser $7,4 \pm 1,65$ ( $p$-valor $<0,001$ ), diferença estatisticamente significante na redução da hemoglobina glicada.

O tempo médio de tratamento realizado pelos pacientes foi de $20,4 \pm 14,48$ meses, variando de 4 a 66 meses e $50 \%$ dos pacientes realizaram até 16 meses de tratamento. Ao analisar a distribuição da frequência na redução da hemoglobina glicada, verificamos que houve queda dos níveis em $81,1 \%$ dos pacientes, sendo que apenas 4,2\% não apresentaram diferença ao comparar a $\mathrm{Hb} 1 \mathrm{Ac}$ inicial com a final e $14,7 \%$ obtiveram incremento após o início do acompanhamento (Tabela 1).

Tabela 1 - Distribuição da frequência da variação da hemoglobina glicada, $n=95$.

\begin{tabular}{lcc}
\hline Variável & $\mathbf{N}$ & $\%$ \\
\hline Variação da hemoglobina glicada & & \\
\hline Incremento & 14 & 14,7 \\
Ausência de variação & 04 & 4,2 \\
Redução entre $0,1-1,0 \%$ & 29 & 30,5 \\
Redução entre 1,1-2,0\% & 24 & 25,3 \\
Redução entre 2,1-3,0\% & 16 & 16,9 \\
Redução > 3,0\% & 08 & 8,4 \\
\hline Total & $\mathbf{9 5}$ & $\mathbf{1 0 0 \%}$ \\
\hline
\end{tabular}

Fonte: Melo Filho PH, 2021.

A classe dos medicamentos dispensados por LME mais utilizada foi a dos análogos de insulina (40\%), seguida pela classe dos inibidores da DDP-4 associados a metformina $(37,9 \%)$; a classe dos inibidores da DDP-4 isoladamente é utilizada por $24,2 \%$ dos pacientes e a classe das glitazonas é usada por $15,8 \%$. Os pacientes também fazem uso de outras medicações que não as fornecidas por LME e entre elas a mais utilizada foi a classe das sulfonilureias $(42,1 \%)$, seguida pela metformina $(28,4 \%)$. Já a classe das insulinas $\mathrm{NPH} /$ Regular e outras classes são utilizadas por $8,4 \%$ e $6,3 \%$ dos pacientes, respectivamente.

Evidenciamos que a distribuição dos pacientes com controle glicêmico adequado é diferente conforme 0 tempo de diagnóstico da doença ( $p$-valor $=0,021$ ). Pacientes com tempo de doença menor que 5 anos têm 3,71 vezes mais chance de ter controle glicêmico adequado se comparado a pacientes com mais de 10 anos de doença IC95\% $(1,23 ; 11,24)$ e pacientes com tempo de doença entre 5 e 10 anos têm 2,94 vezes mais chance de ter controle glicêmico adequado se comparado a pacientes com mais de 10 anos de doença IC95\% $(1,002 ; 8,622)$ (Tabela 2). Não houve indícios de diferenças estatisticamente significantes no controle glicêmico de acordo com o sexo, idade e demais variáveis analisadas.

Tabela 2 - Distribuição do controle glicêmico segundo algumas variáveis de pacientes diabéticos do tipo 2, $\mathrm{n}=95$.

\begin{tabular}{|c|c|c|c|c|c|}
\hline \multirow{2}{*}{ Variável } & \multicolumn{2}{|c|}{ Controle Glicêmico } & \multirow{2}{*}{ p-valor } & \multirow{2}{*}{ OR } & \multirow{2}{*}{ IC } \\
\hline & Adequado & Inadequado & & & \\
\hline \multicolumn{6}{|l|}{ Tempo de Diagnóstico } \\
\hline Menor que 5 anos & 13 & 6 & & 3,71 & $(1,23 ; 11,24)$ \\
\hline Entre 5 e 10 anos & 12 & 7 & 0,021 & 2,94 & $(1,002 ; 8,622)$ \\
\hline Maior que 10 anos & 21 & 36 & & - & - \\
\hline
\end{tabular}

Fonte: Melo Filho PH, 2021. 


\section{DISCUSSÃO}

O diabetes é uma das doenças crônicas necessita de maior nível de atenção e cuidado, visto que suas complicações agudas e crônicas implicam em importante morbidade, além de altas taxas de hospitalizações e de mortalidade, promovendo significativos gastos econômicos e prejuízos sociais em decorrência da redução da sobrevida da população com diabetes. Vale ressaltar que o motivo de boa parte da população diabética desconhecer o diagnóstico se deve, principalmente, pelo fato de a doença ser oligossintomática e de início e progressão dos sintomas serem insidiosos, além do baixo desempenho dos sistemas de saúde e da escassa conscientização sobre diabetes entre a população geral e os profissionais de saúde.

Em virtude da crescente ocorrência de complicações crônicas associadas ao diabetes e dos elevados custos diretos e indiretos ao sistema de saúde, optou-se pela realização do presente estudo e, que assim como em outros da mesma natureza, pode-se afirmar que a maioria dos pacientes avaliados se encontra fora das metas definidas pela SBD para controle clínico da doença, sendo que a frequência inicial de controle glicêmico adequado ( $\mathrm{Hb} 1 \mathrm{Ac} \leq 7)$ nessa avaliação foi de $15 \%$ e após a ampliação do arsenal terapêutico passou a ser de $48 \%$ dos pacientes ( $p$-valor $<0,001$ ), número ainda inferior ao adequado, porém com clara relação de melhora.

Portanto, percebe-se que os pacientes diabéticos do tipo 2 acompanhados no ambulatório de Endocrinologia de um hospital terciário do Nordeste apresentaram melhor controle da doença após a introdução dos antidiabéticos orais e insulinas dispensadas pela farmácia de medicamentos especializados do Piauí. Deste modo, há evidências de que após a disponibilização de uma maior variedade de medicamentos para o tratamento do diabetes houve aumento no número de pacientes com controle glicêmico adequado.

De uma forma geral, a frequência de pacientes com controle adequado encontrada, após o acompanhamento, se assemelha à de outras pesquisas descritas na literatura, como Li J, et al. (2018), estudo realizado em um centro terciário de diabetes em Ningbo na China, no qual a prevalência do bom controle glicêmico foi de 50,3\%, e o de Formiga F, et al. (2017), conduzido na Espanha com uma população de 7.269 pacientes idosos com média de idade 73,4 anos e $48,4 \%$ do sexo masculino, onde a $43,2 \%$ deles apresentavam controle adequado. Ambos os estudos com população composta apenas por pacientes com diagnóstico de DM tipo 2.

Já no estudo de Afroz A, et al (2019), realizado em Bangladesh com objetivo de determinar o controle glicêmico entre pessoas com DM tipo 2 e tempo médio de diagnóstico de 10 anos, cerca de $82 \%$ dos participantes tinham controle glicêmico inadequado e $54,7 \%$ tinham controle muito ruim ( $\mathrm{HbA} 1 \mathrm{c} \geq 9 \%$ ). Fatores como baixa escolaridade, residência rural, hábitos alimentares pouco saudáveis, uso de insulina e consultas infrequentes foram associados a controles inadequados. Essa prevalência se assemelha a encontrada em nosso estudo no início do acompanhamento, momento no qual o arsenal terapêutico era reduzido.

Outros estudos realizados apenas com pacientes diabéticos do tipo 2, como o de Atif M, et al. (2019), conduzido com pacientes idosos em Lahore, Paquistão, que mostrou $77,8 \%$ dos pacientes apresentava hemoglobina glicada acima do nível-alvo, o de Patrick NB, et al. (2020) realizado com pacientes atendidos no ambulatório do Hospital Regional de Referência de Mbarara em Uganda, mostrou uma prevalência de 84,3\% de controle glicêmico ruim, e o de Alramadan MJ, et al. (2018), na Arábia Saudita, que descreveu cerca de três quartos dos participantes com controle glicêmico inadequado, sendo 21,7\% tiveram controle parcial (7$7,9 \%$ ) e $54,2 \%$ tinham controle insatisfatório ( $\geq 8 \%$ )

A variação de resultados entre os estudos pode ser explicada devido a diferenças nas populações avaliadas, nos métodos de coleta de dados, no método de medição da $\mathrm{HbA} 1 \mathrm{c}$ e nas definições de pontos de corte de $\mathrm{HbA} 1 \mathrm{c}$ adequados para controle da glicemia. No entanto, nota-se uma prevalência mais elevada de inadequado controle glicêmico em países economicamente desfavorecidos, sendo que os níveis encontrados em nosso estudo se assemelham mais aos de países como China e Espanha. 
Ao avaliar a hemoglobina glicada em dois momentos distinto, o primeiro antes de iniciar o tratamento especializado com as medicações disponibilizadas pela farmácia de alto custo e o segundo no ato da coleta dos dados, em nossa avaliação, a princípio, a média de HbA1c dos pacientes era de 8,6 $\pm 1,60$ e após passou a ser 7,4 $\pm 1,65$ ( $p$-valor < 0,001), diferença estatisticamente significante, ou seja, houve uma redução da hemoglobina glicada após a ampliação do acesso aos demais medicamentos para o tratamento com antidiabéticos orais e demais análogos de insulina, embora ainda com valores fora das metas definidas pela SBD. No entanto, vale ressaltar que em $81,1 \%$ dos pacientes avaliados foi evidenciada redução dos níveis de hemoglobina glicada, fator associado a melhora dos parâmetros metabólicos.

Em estudo realizado por Duarte FG, et al. (2019), com uma população de 9418 pacientes com diabetes tipo 2, sendo 5692 brasileiros e 3726 venezuelanos, foi observado que a média da hemoglobina glicada foi maior entre as mulheres $(8,8 \%)$ do que entre os homens $(8,6 \%)$. Outros estudos mantêm esse média de $\mathrm{HbA1c}$ acima da encontrada em nosso estudo no segundo momento, mas semelhante a observada no primeiro momento. Alramandan $\mathrm{MJ}$, et al. (2018), encontrou em seu estudo média de $8,5 \%( \pm 1,9 \%)$, e Caprnda M, et al (2017) em um estudo com 99 pacientes (46 homens, 53 mulheres) e duração média do diagnóstico de 15 anos, o nível de hemoglobina glicada foi de 9,05\% $\pm 2,54 \%$.

Em um estudo randomizado brasileiro realizado por Trevisan DD, et al. (2019), com o objetivo de avaliar os efeitos de uma intervenção de intenção no controle glicêmico em pacientes com diabetes mellitus tipo 2 tratados no nível de atenção primária, o nível médio de A1C foi de $7,2 \%$ antes da intervenção. Tal estudo foi o que mais se assemelhou aos resultados encontrados no segundo momento de observação do nosso trabalho.

O estudo possui limitações em razão da coleta de informações ter ocorrido através de uma revisão de prontuários, pois não se pode descartar imprecisões e falta de alguns dados obtidos, em razão da ausência de controle sobre a coleta de informações, fato este que pode ter restringido o tamanho de nossa amostra. No entanto, os resultados aqui apresentados levantam questões que merecem consideração futura. Os dados proporcionados são compatíveis aos encontrados na literatura e inéditos no âmbito regional. Portanto, faz-se necessário aumentar o orçamento dedicado a todos os aspectos do cuidado com o diabetes, melhorando a acessibilidade e disponibilidade dos medicamentos e insumos essenciais, como medicações e tecnologias inovadoras, ampliando o arsenal terapêutico disponível no SUS.

\section{CONCLUSÃO}

Com base nos resultados obtidos, podemos observar que foi possível perceber um melhor controle glicêmico dos pacientes quando comparado o início do processo ao momento posterior, após o uso das medicações especializadas, evidenciado tanto pelo aumento do número de pacientes com hemoglobina glicada $\leq 7$, como pela redução da média de $\mathrm{Hb} 1 \mathrm{Ac}$ quando confrontadas. Nota-se, portanto, a importância do amplo acesso às mais diversas classes de medicamentos para o tratamento do diabetes tipo 2 no âmbito do SUS, a fim de melhorar o controle glicêmico desses pacientes e reduzir desfechos desfavoráveis.

\section{REFERÊNCIAS}

1. AFROZ A, et al. Glycaemic Control for People with Type 2 Diabetes Mellitus in Bangladesh - An urgent need for optimization of management plan. Scientific Reports, 2019; 15:9.

2. ALRAMADAN MJ, et al. Glycaemic control for people with type 2 diabetes in Saudi Arabia - an urgent need for a review of management plan. BMC Endocrine Disorders, 2018; 18:62.

3. AMERICAN DIABETES ASSOCIATION (ADA). Standards of Medical Care in Diabetes. 2020. Disponível em: https://care.diabetesjournals.org/content/43/Supplement_1. Acessado em: 23 de setembro de 2021.

4. ATIF M, et al. Extent and Predictors of Poor Glycaemic Control among Elderly Pakistani Patients with Type 2 Diabetes Mellitus: A Multi-Centre Cross-Sectional Study. Medicina, 2019; 55:21.

5. BAHIA LR, et al. The costs of type 2 diabetes mellitus outpatient care in the Brazilian public health system. Value Health, 2011; 14: 137-40.

6. BERTOLUCI MC, et al. Diabetes and cardiovascular disease: from evidence to clinical practice - position statement 2014 of Brazilian. Diabetes Society Diabetology \& Metabolic Syndrome, 2014; 6: 58. 
7. BRASIL. Lei no 8080 , de 19 de setembro de 1990. Disponível em: http://www.planalto.gov.br/ccivil_03/leis//8080.htm. Acessado em: 23 de setembro de 2021.

8. BRASIL. Lei no 8142, de 28 de dezembro de 1990. Disponível em: http://www.planalto.gov.br/ccivil_03/leis/l8142.htm. Acessado em: 23 de setembro de 2021.

9. BRASIL. Portaria no 371/GM, de 04 de março de 2002. Disponível em: https://bvsms.saude.gov.br/bvs/saudelegis/gm/2002/prt0371_04_03_2002_rep.html. Acessado em: 23 de setembro de 2021.

10. BRASIL. Portaria no 1.096 , de 25 de novembro de 2020. Disponível em: https://www.in.gov.br/en/web/dou/-/portarian-1.096-de-25-de-novembro-de-2020-*-293247719. Acessado em: 23 de setembro de 2021.

11. CAPRNDA M, et al. Glycemic Variability and Vascular Complications in Patients with Type 2 Diabetes Mellitus. Folia Medica, 2017; 59:3.

12. DIABETES CONTROL AND COMPLICATIONS TRIAL (DCCT). The effect of intensive treatment of intensive treatment of diabetes on the development and progression of long-term complications in insulin-dependent diabetes mellitus New England Journal of Medicine, 1993; 329: 977-86.

13. DUARTE FG, et al. Sex differences and correlates of poor glycaemic control in type 2 diabetes: a cross-sectional study in Brazil and Venezuela. BMJ Open, 2019; 9:3.

14. FORMIGA F, et al. Inadequate Glycaemic Control and Therapeutic Management of Adults over 65 Years Old with Type 2 Diabetes Mellitus in Spain. The Journal of Nutrition, Health \& Aging, 2017; 21:10.

15. INTERNATIONAL DIABETES FEDERATION (IDF). IDF Diabetes Atlas, 9nd ed. 2019. Disponível em: https://www.diabetesatlas.org/en/. Acessado em: 23 de setembro de 2021.

16. LI J, et al. Glycaemic control in type 2 diabetes patients and its predictors: a retrospective database study at a tertiary care diabetes centre in Ningbo, China. BMJ Open, 2018; 8:3.

17. PATRICK NB, et al. Poor Glycemic Control and the Contributing Factors Among Type 2 Diabetes Mellitus Patients Attending Outpatient Diabetes Clinic at Mbarara Regional Referral Hospital, Uganda. Diabetes, Metabolic Syndrome and Obesity: Targets and Therapy, 2021; 14.

18. PIAUÍ. Assistência Farmacêutica do Piauí - Lista de medicamentos entregues na farmácia. 2020. Disponível em: https://sites.google.com/view/farmaciaespecializada/documentos/listademedicamentos. Acessado em: 28 de setembro de 2021.

19. SOCIEDADE BRASILEIRA DE DIABETES (SDB). Diretrizes. 2021. Disponível em: https://diretriz.diabetes.org.br/. Acessado em: 23 de setembro de 2021.

20. TREVISAN DD, et al. Effect of an 'implementation intention' intervention on adherence to oral anti-diabetic medication in Brazilians with type 2 diabetes. Patient Education and Counseling, 2019; 103:3.

21. UNITED KINGDOM PROSPECTIVE DIABETES STUDY. Intensive blood glucose control with sulphonylureas or insulin compared with conventional treatment and risk of complications in patients with type 2 diabetes. The Lancet, 1998; 352: 837-53.

22. VILAR L. Endocrinologia Clínica. 7ª̣ edição. Rio de Janeiro: Guanabara Koogan, 2021; 665p. 